\title{
APPLICATIONS OF X-BAND TECHNOLOGY IN MEDICAL ACCELERATORS
}

\author{
S. M. HANNA ${ }^{+}$, Siemens Medical Systems-OCS, Concord, CA
}

\section{Abstract}

Most radiation therapy machines are based on microwave linear accelerators. The majority of medical accelerators use frequencies in the S-band range. Having a compact accelerator allows for a wide range of treatments. The size and weight of the accelerator is substantially reduced if a higher frequency is used. X-band frequencies are suitable for such applications. The X-band accelerator technology has been used in high-energy as well as industrial applications. In the radiation therapy field, it is already implemented in some machines. The Mobetron [1], an Intra-Operative Radiation Therapy (IORT) treatment system is one example. Another example is the Stereotactic Radiosurgery machine, the CyberKnife [2]. The compactness of these machines required the use of an $\mathrm{X}$-band accelerator. The basis for choosing the X-band technology in some of the medical machines is analysed. A review of the exiting medical applications is included. We also discuss the availability of other X-band components in the machine, including high-power RF sources.

\section{X-BAND ACCELERATOR TECHNOLOGY}

X-band accelerator development has gained great momentum in the last ten years, motivated by the need for high-gradient accelerators for the future linear colliders in high-energy physics research [3]. Design of accelerator cells [4], manufacturing [5], and characterization technique [6] have been developed.

In the industrial [7] and medical [1,2] fields, there are obvious advantages for using frequencies higher than the $\mathrm{S}$-band range that is prevalent in these applications. The dominant factor is the compactness of the accelerator with the attendant reduction in weight. Consequently, the linac motion can be precisely controlled. By implementing the X-band technology, one can use a shorter length for a given power to achieve a certain electron beam energy. The reasons for this are two-fold. First, the shunt impedance per unit length is higher than that of S-band. Second, the maximum permissible electric field strength is also higher. The frequency dependence of these accelerator parameters is listed in Table 1.

+Email: shanna@smsocs.com
Table 1: Frequency Dependence of Accelerator Parameters

\begin{tabular}{|l|c|}
\hline Parameter & $\begin{array}{c}\text { Frequency } \\
\text { Dependence }\end{array}$ \\
\hline Shunt impedance per unit length (r) & $f^{1 / 2}$ \\
\hline $\begin{array}{l}\text { Maximum permissible electric field } \\
\text { strength }\end{array}$ & $f^{1 / 2}$ \\
\hline RF loss factor (Q) & $f^{-1 / 2}$ \\
\hline $\begin{array}{l}\text { Power dissipation capability of } \\
\text { accelerator structure }\end{array}$ & $f^{-1}$ \\
\hline
\end{tabular}

Certainly there are some trade-off factors to be considered with the use of X-band. These include the lower Q and lower power dissipation capability of the accelerator structure, as indicated in Table 1. These can be overcome by careful design of the cooling system. In addition, a better surface finish and tighter dimension tolerances are required.

\section{MEDICAL APPLICATIONS}

Currently, there are two different radiation therapy applications that use the X-band technology.

\subsection{Inter-Operative Radiation Therapy}

The Inter-Operative Radiation Therapy (IORT) technique basically refers to the delivery of radiation during an operation using an electron beam. This technique of radiation therapy has been proven to increase the survival rates in certain cancer cases. Conventional radiation therapy machines must be housed in special shielded rooms, requiring the patient to be moved from the operating room. A mobile radiation therapy machine, the Mobetron [1], engineered and developed by Intraop, has met the need for a self-shielded machine. Since the unit is mobile, it can be easily positioned in an operating room to treat cancerous growths with good precision during surgery, thus, increasing the effectiveness of radiation doses to tumors while reducing the dose to surrounding healthy tissue.

The design approach for the Mobetron exploits some of the unique advantages of using the X-band technology mentioned above. The Mobetron treatment head houses the X-band linac, the magnetron, the pulse transformer, RF loads, the circulator, and other RF circuitry. 
The linac is composed of two colinear accelerator sections. The energy of the electron beam is changed by varying the power to the second section. This is done through a phasing technique using movable shorts driven by motors controlled by the energy control system. Thus, the Mobetron can deliver multiple electron energies, namely, $4 \mathrm{MeV}, 6 \mathrm{MeV}, 9 \mathrm{MeV}$, and $12 \mathrm{MeV}$ at two dose rate settings $(250 \mathrm{cGy} / \mathrm{min}$ and $1000 \mathrm{cGy} / \mathrm{min})$. The electron beam exits the accelerator through a thin titanium vacuum window.

Siemens Medical Systems, Inc. - Oncology Care Systems (OCS), is manufacturing the Mobetron as well as marketing it in collaboration with Intraop Medical, Inc.

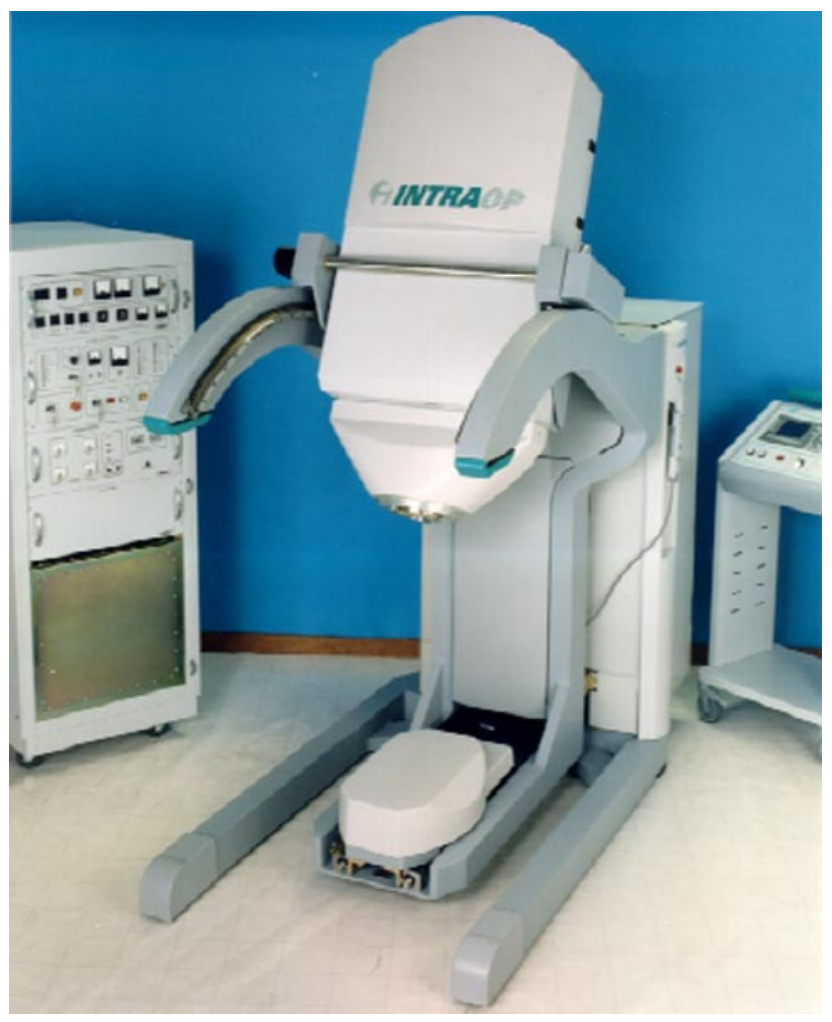

Figure 1: The Mobetron IORT system.

\subsection{Stereotactic Radiosurgery}

Another important application for the X-band technology is stereotactic radiosurgery. Accuray Oncology has developed the CyberKnife [2]. This machine uses an image-guided robotic system to precisely deliver an X-ray beam to focal lesions. It integrates treatment planning, imaging, and delivery components, all of which are controlled by a Workstation. The CyberKnife uses a compact X-band $6 \mathrm{MeV}$ Linac operating at $9.3 \mathrm{GHz}$ and weighing $285 \mathrm{lbs}$. The relatively lightweight Linac makes it possible for the robotic arm carrying it to be accurately positioned. Fig.2 shows the CyberKnife [8].

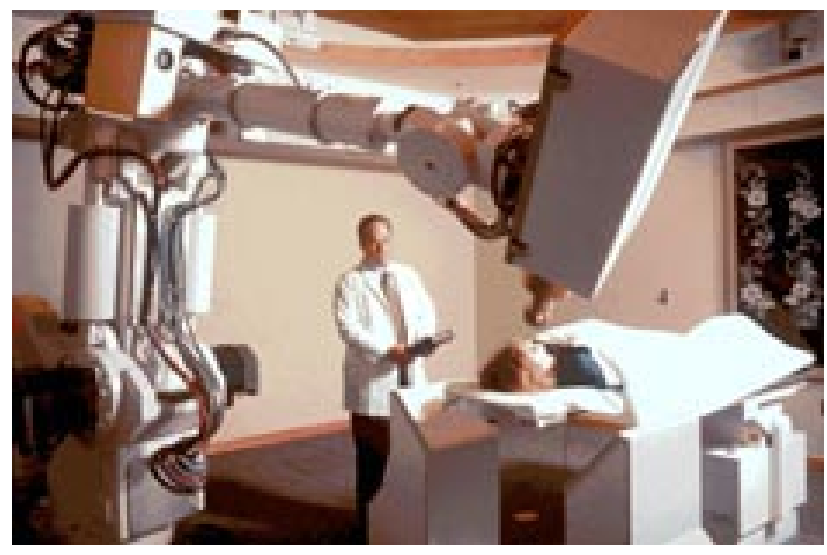

Figure 2: The CyberKnife stereotactic radiotherapy system.

\section{HIGH POWER MICROWAVE SOURCES}

The S-band has been the prevailing frequency range for linacs in medical and industrial applications. This is mainly attributed to the availability of high power microwave sources in this frequency range. Recently there have been efforts in the area of development for the $\mathrm{X}$-band sources.

The RF high-power source for both the Mobetron and the CyberKnife linacs is the 1.5 MW pulsed magnetron (PM-1100X). The California Tube Laboratory, Inc, builds this tunable magnetron; it is shown in Fig. 3. Other efforts for developing $\mathrm{X}$-band sources include the tunable $1.5 \mathrm{MW} \mathrm{X}$-band currently in development at EEV and the 4 MW klystron by Mitsubishi [9].

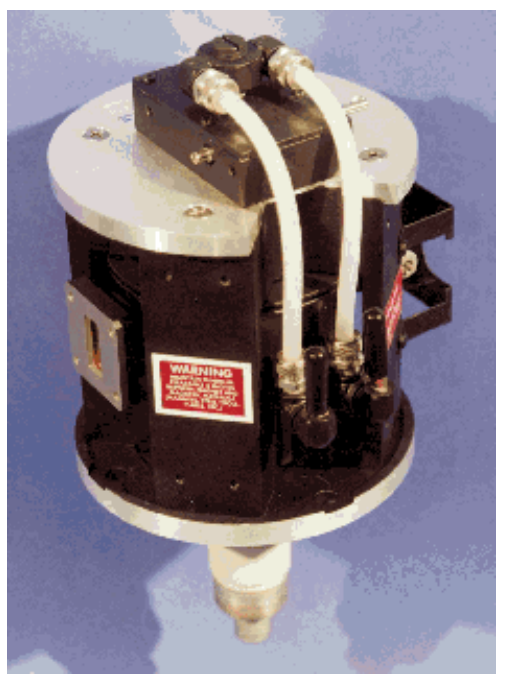

Figure 3: The CTL 1.5 MW X-band magnetron. 


\section{DISCUSSION}

Recent X-band research and development for linear collider projects have been providing an impetus for the development of X-band accelerators and RF sources. Klystrons having output power of $50 \mathrm{MW}$ and higher have been developed at SLAC. However, for the medical linacs, the need still exists for the development of X-band sources of a few MW of peak power.

\section{REFERENCES}

[1] M. L. Meurk et al., "The Mobetron: A New Concept for Intraoperative Radiotherapy", $6^{\text {th }}$ International IORT Symposium, San Francisco, CA (1996).

[2] S. G. Chenery et al., "The CyberKnife: Beta System Description and Initial Clinical Results", Journal of Radiosurgery, Vol. 1, No.4, (1998).

[3] J. W. Wang et al. "Accelerator Structures R\&D for Linear Colliders", This Conference (1999).

[4] J. Klingmann et al., "Fabrication of DDS-3, an 11.4 GHz DampedDetuned Structure", This Conference (1999).

[5] Z. Li, "RDDS Cell Design and Optimisation for the NLC Linac", This Conference (1999).

[6] S.M. Hanna et al., "Development of Characterisation Techniques for X-Band Accelerator Structures", Proc. Of the 1997 IEEE PAC (1997).

[7] R. G. Schonberg et al., "Portable X-Band Linear Accelerator System”, IEEE Trans. On Nuc. Sc., Vol. NS-32, No. 5 (1985).

[8] W. T. Main, Private Communication.

[9] K. Hayashi et al., "X-band High Power Pulsed Klystron", Proc. Of the 1988 Linear Accelerator Conf. , pp 562-564 (1988). 